MATEC Web of Conferences 13, 02006 (2014)

DOI: $10.1051 /$ matecconf/ 20141302006

(C) Owned by the authors, published by EDP Sciences, 2014

\title{
Characterization of Water in Diesel Emulsion
}

\author{
Z. A. Abdul Karim ${ }^{1, a}$, Mohammed Yahaya Khan ${ }^{1}$, A. Rashid A. Aziz ${ }^{1}$ and Isa M Tan ${ }^{2}$ \\ ${ }^{1}$ Department of Mechanical Engineering, Universiti Teknologi Petronas, Malaysia \\ ${ }^{2}$ Department of Fundamental \& Applied Science, University Teknologi Petronas, Malaysia
}

\begin{abstract}
Water in diesel emulsion, as an option fuel, has potential to simultaneously reduce the formation of both nitrogen oxides and particulate matters in diesel engine. However, the capability of this fuel strongly dependent on the type of emulsion, stability of the emulsified fuel and the physio-chemical properties. In this study, water in diesel emulsion fuels of $5 \%, 10 \%, 20 \%$, water by volume was prepared by a mechanical homogenizer. Physical and chemical properties of the emulsion were examined as these properties could influence the spray characteristics of the emulsions which significantly affect the ignition delay and flame propagation. Density and viscosity was found to be higher for all of the water in diesel emulsion than pure diesel at all measured temperatures whereas the carbon contents for water in diesel emulsion with $10 \%$ and $20 \%$ water were low. Droplet size of the emulsion was found to be less than $2 \mu \mathrm{m}$. The actual water content in the emulsified fuel was found to be lesser than the mixed amount.
\end{abstract}

Keywords: Emulsified fuel, homogeniser, surfactant; droplet size, emulsion properties.

\section{Introduction}

In spite of preferable advantages of diesel engines, they are one of the major pollution contributors to present time. Such primary pollutants exhausted from diesel engines are particulate matters (PM), nitrogen oxides $(\mathrm{NOx})$, Sulphur oxides $\left(\mathrm{SO}_{\mathrm{X}}\right)$, unburnt hydrocarbon $(\mathrm{HC})$, carbon monoxide $(\mathrm{CO})$, and carbon dioxide $\left(\mathrm{CO}_{2}\right)$ [1]. Emulsion of diesel and water are often promoted as being able to overcome the difficulty of simultaneously reducing emissions of both $\mathrm{NO}_{\mathrm{X}}$ and $\mathrm{PM}$ from the diesel engines [2]. The main mechanism causing the reduction in NOx emissions seems to be the decrease in the temperature of the combustion products as a result of vaporisation of the liquid water and consequent dilution of the gas phase species. As for PM emissions, the presence of water during the intensive formation of soot particles seems to reduce the rate of formation of soot particles and enhance their burnout by increased concentration of oxidation species such as $\mathrm{OH}$ [3]. The aim of the present study is to obtain stable water in diesel emulsion (WiDE) which is stabilized with surfactants, in order to reduce operating costs, emissions and fuel consumptions of any internal-combustion engine. Primary step before using WiDE as a fuel is to characterize their properties as it will influence certain operating parameter of the engine such as injection timing and mixing process [4]. Besides that, it was found that physical and chemical kinetics of the combustion is influenced by the presence of water vapour in fuel [5].

\footnotetext{
${ }^{a}$ Corresponding author : ambri@petronas.com.my
}

This is an Open Access article distributed under the terms of the Creative Commons Attribution License 2.0, which permits unrestricted use, distribution, and reproduction in any medium, provided the original work is properly cited. 


\section{Methodology}

Two-phase water in diesel emulsion (WiDE) as shown in Figure 1 was prepared using mechanical homogenizer. Commercially available surfactant, commercial diesel, and distilled water were used for the emulsion. Three blends of WiDE with 5\%,10\%,20\%, water by volume were prepared.

Anton Paar Microviscometer Lovis 2000ME and density meter DMA 4500M were used to measure the viscosity and density of WiDE and diesel at different temperatures. Calorific values of the fuels were obtained using Leco AC-350 bomb calorimeter which was calibrated using standard benzoic acid as standard. Water droplet and distribution was captured by using MEIJI-ML 9300 and measurements were made by using software Toupview-3.0. Chemical characteristics were analysed using CHNS analyser. Stability of the WiDE was visually observed. METTLER TOLEDO T70 auto titrator was used to ascertain the actual water content in WiDE upon homogenising. Hydranal composite- 5 and methanol rapid were used as reagent and solvent respectively.

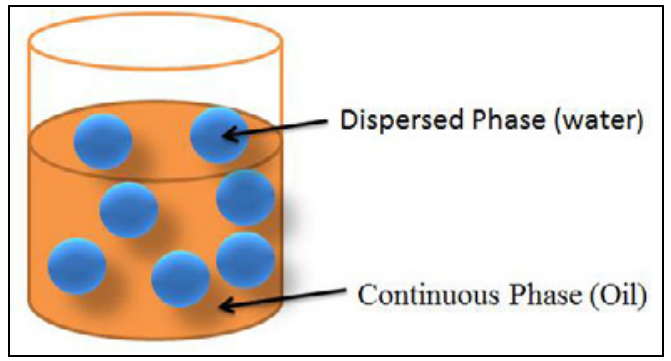

Figure 1. Concept of two-phase Water-in-oil emulsion [6]

\section{Results and Discussions}

\subsection{Stability of WiDE}

The most important factor in the preparation of emulsions is the selection of suitable surfactant or blend of surfactants which can satisfactorily emulsify the chosen ingredients at a specific temperature. Emulsification is greatly influenced by hydrophile-lipophile balance (HLB) of any surfactant/ emulsifier [7]. Percentage of water in the emulsion, stirring intensity, stirring duration, emulsifying temperature and operational pressure are other parameters of interest that affect the stability of an emulsion. The WiDE prepared for the current study was observed for more than 90 days since it was emulsified and found to be stable as shown in Figure 2. It was noted that, the larger the amount of water content in the emulsion, the brighter milky emulsion is produced.

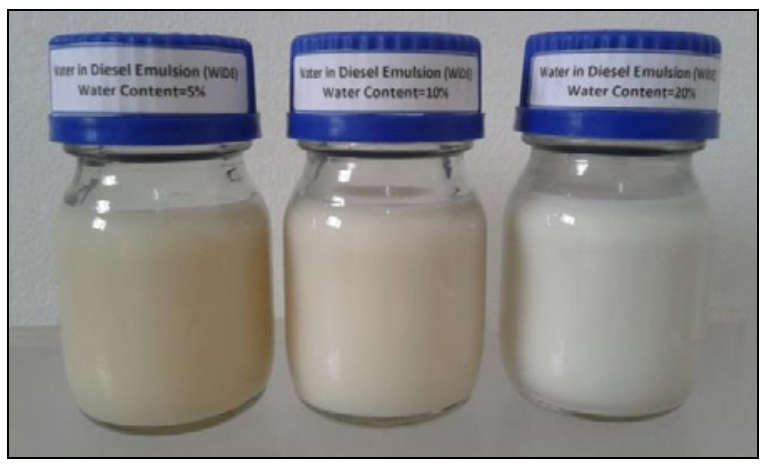

Figure 2. Stable WiDE samples with 5\%, 10\% and 20\% water 


\subsection{Water content of WiDE}

The presence of water in WiDE is a key issue to be addressed since it will affect the ignition delay timing [8, 9]. Karl Fischer titration (volumetric) principle was used in determining the actual water content of the prepared WiDE samples.

Table 1. Water content in WiDE

\begin{tabular}{|c|c|c|c|}
\hline Sample & $\begin{array}{c}\text { Expected water content } \\
(\mathbf{\%})\end{array}$ & $\begin{array}{c}\text { Actual water content } \\
(\mathbf{\%})\end{array}$ & $\begin{array}{c}\text { Difference in } \\
\text { water content }\end{array}$ \\
\hline WiDE-5 & 5 & 3.323 & 1.677 \\
\hline WiDE-10 & 10 & 6.408 & 3.592 \\
\hline WiDE-20 & 20 & 14.681 & 5.319 \\
\hline
\end{tabular}

Contradictorily, water content was found to be lesser upon mixing in all WiDE samples as summarised in Table 1. In order to affirm the results, each test was repeated thrice and average values were tabulated. The probable reason for lesser water content in the prepared WiDE is due to evaporation of water during homogenising. During the process to break the droplet size into micro size level, the emulsion was made to pass through a stator and a rotor spaced at 50 microns gap with a rotating speed of $3000 \mathrm{rpm}$. Hence water was subjected to very high shearing force at elevated temperature $\left(85^{\circ} \mathrm{C}-90^{\circ} \mathrm{C}\right)$ and pressure $(1-2$ bar) leading to cavitation that caused water to evaporate. This reveals that production method is also one of the important criteria to be considered while producing WiDE to maintain the required water level in the emulsion.

\subsection{Density and Viscosity}

It is evident that the density of the emulsion increases with increase in the amount of water in the mixture. This is attributed to the higher density of water that is being added to diesel fuel which is of lower density. Densities for diesel and the three WiDE samples measured at $20^{\circ} \mathrm{C}$ to $50^{\circ} \mathrm{C}$ are plotted in Figure 3. WiDE-20 has 3.46\% higher density than diesel at $50^{\circ} \mathrm{C}$. Difference in density level between WiDE-5 and WiDE-10 is between 0.54 to $0.75 \%$ and it was around $1.8 \%$ to $1.9 \%$ in case of WiDE-10 and WiDE-20.

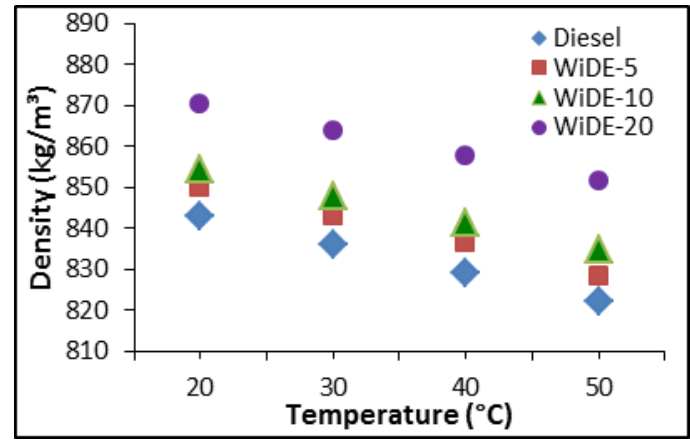

Figure 3. Density of Diesel and WiDE samples

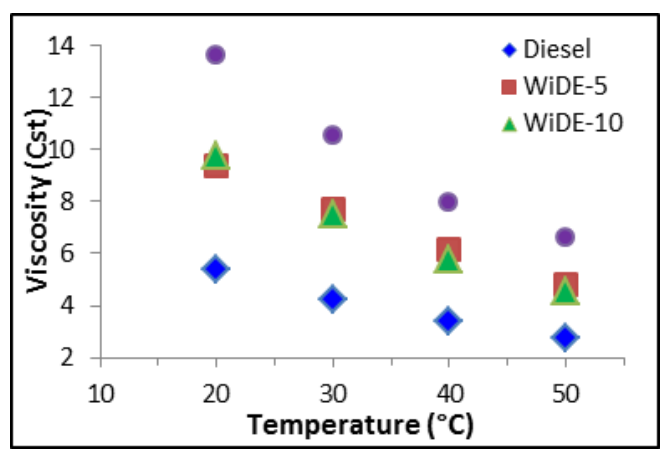

Figure 4. Viscosity of Diesel and WiDE samples

Viscometer was used to find viscosity of WiDE and diesel for the temperature range as shown in Figure 4 . The viscosity of WiDE-20 is $58 \%$ higher than diesel at $50^{\circ} \mathrm{C}$. The difference in viscosity between WiDE-5 and WiDE-10 is small while in case of WiDE-10 and WiDE-20 it was around 28.28 to $31.16 \%$. Both density and viscosity variation between WiDE-5 and WiDE-10 are insignificant and is due to the reduction in the actual water content in the mixture which was not maintained. 


\subsection{Calorific Value}

The presence of water leads to lower heating value of WiDE and can be attributed to slight reduction in engine power when used as fuel. The gross calorific value of WiDE was decreased with increase water content as shown in Table 2.

Table 2. Calorific Value of WiDE and Diesel

\begin{tabular}{|c|c|}
\hline Sample-ID & Gross Calorific Value ( J/g) \\
\hline Diesel & 42600 \\
\hline WiDE-5 & 37460 \\
\hline WiDE-10 & 36942 \\
\hline WiDE-20 & 32481 \\
\hline
\end{tabular}

\subsection{CHNS Analysis}

Carbon content was higher than diesel for WiDE with $5 \%$ water which can be attributed to the influence of surfactant along with lesser water. Carbon for WiDE with $10 \%$ and $20 \%$ water were less compared to diesel. Hydrogen presence was higher than pure diesel for WiDE with 5\% and 10\% water. Whereas, sulphur was found to be higher for all three WiDE samples, as shown in Table 3. Surfactants used should easily burn with no soot and free of sulphur and nitrogen [10].

Table 3. CHNS values of diesel and WiDE with $5 \%, 10 \%$ and $20 \%$ water

\begin{tabular}{|c|c|c|c|c|}
\hline Sample ID & Carbon\% [w/w] & Hydrogen \%[w/w] & Nitrogen \%[w/w] & Sulphur \%[w/w] \\
\hline Diesel & 81.38 & 11.86 & 0.1013 & 0.1257 \\
\hline WiDE-5 & 86.32 & 16.416 & 0.1123 & 0.821 \\
\hline WiDE-10 & 78.65 & 12.403 & 0.193 & 0.6793 \\
\hline WiDE-20 & 68.94 & 9.734 & 0.221 & 0.4793 \\
\hline
\end{tabular}

\subsection{Water distribution and Droplet Diameter}

The distribution of water in WiDE of 5\%,10\% and 20\% are shown in Figure 5 (a) to (c), respectively. The images were captured by optical microscope with $400 \mathrm{X}$ magnification. It is known that the droplet diameter of the micro-emulsions should not vary beyond 0.1 to $20 \mu \mathrm{m}$. The smaller droplets tend to evaporate more quickly and form a better air-fuel mixture, hence the reduction in soot when the mixture burns [11-13]. Images show that mean droplet diameters are in the acceptable range for all the three WiDE samples.

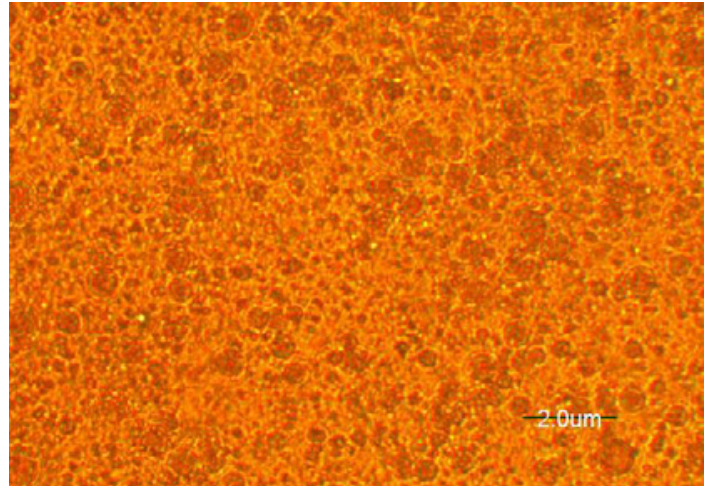

(a) WiDE-5

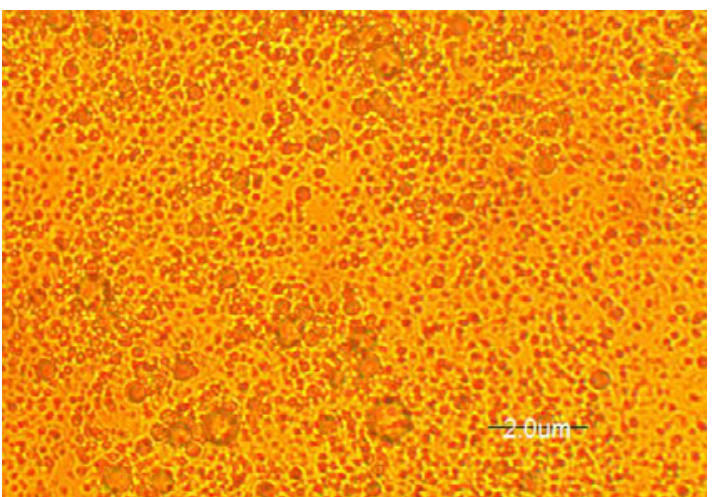

(b) WiDE-10 


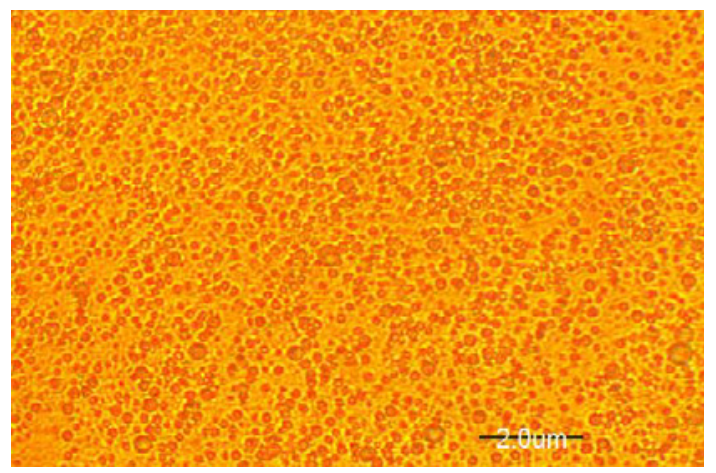

(c) WiDE-20

Figure 5. Image of WiDE samples with 400X magnification

\section{Conclusion}

Two-phase stable water in diesel emulsion (WiDE) with 5\%,10\% and $20 \%$ water were successfully prepared using mechanical homogenizer and their physical and chemical properties were examined and discussed in detail. Actual water contents in the emulsified fuels were found to be lesser than the mixed amount upon homogenisation. Droplet sizes of less than $2 \mu \mathrm{m}$ were achieved. Density and viscosity was found to be higher for all WiDE samples as compared to diesel at all measured temperatures whereas the carbon contents for WiDE with $10 \%$ and $20 \%$ water were low. Further investigations are needed to determine other influential properties such as cetane number and adiabatic flame temperature. In addition, developed WiDE samples have to be tested on the engine to investigate the effects on the combustion characteristics, emission formation processes and engine behaviours. Also, it is pertinent to determine the pollution formation suppression capability of the emulsified fuels by in-depth combustion characteristics analysis.

\section{Acknowledgement}

The authors thank Universiti Teknologi PETRONAS for the financial and facility support for the study. SIT Schiffs \& Industrie Technik (M) Sdn Bhd is also highly acknowledged for preparing and providing the WiDE samples.

\section{References}

[1] C.Y Lin, K.H Wang, Fuel 82, 1367-1375 (2003).

[2] E. A. Fahd ,Y. Wenming, P.S. Lee, S.K. Chou, C.R. Yap, Appl Energy 102, 1042-1049 (2013).

[3] T. Xuan-Thien, G. Jamil, 5th Asia Pacific Conference on Combustion, (2005).

[4] O. Armas, R. Ballesteros, F.J. Martos, J.R. Agudelo, Fuel 84, 1011-1018 (2005).

[5] A. Alahmer, Energy Conversion and Management 73, 361-369 (2013).

[6] M. Yahaya Khan, Z. A. Abdul Karim, F.Y. Hagos, A. R. A. Aziz, I. M. Tan, TSWJ (2014).

[7] M.J. Rosen, Surfactants and interfacial phenomenon. Wiley; (2004).

[8] I. Jeong, K.H Lee, J. Kim, J Mech Sci and Tech 22, 148-156, 2008.

[9] A. Subramanian, Energy Conversion and Management 52, 849-857 (2011).

[10] Y. Zeng and C.F. Lee, Proc. of the Combustion Institute. 31, 2185-2193 (2007).

[11] K.C. Tsao, X. Yong, SAE, 891883 (1989).

[12] T.Kadota, H.Yamasaki, Prog. Energy Combust. Sci. 28, 385-404 (2002).

[13] Y.Morozumi, Y. Saito, Energy Fuels 24, 1854-1859 (2010). 